\title{
Three-Axis Coil Probe Dimensions and Uncertainties During Measurement of Magnetic Fields from Appliances
}

\section{Martin Misakian and Charles Fenimore}

National Institute of Standards and Technology, Gaithersburg, MD 20899-0001
Comparisons are made between the average magnetic flux densicy for a threeaxis circular coil probe and the flux density at the center of the probe. The results, which are detcrmined assuming a dipolc magnctic field, provide information on the uncertainty associated with medsurements of magnetic fields from some electrical appliances and other electrical equipment. The present investigation extends an carlicr treat- ment of the problem, which Jid not consider all orientations of the probe. A more comprehensive examination of the probicm ficaves unchanged the conclusions reached previously.

Key words: appliancc; coil probe; mag. nctic ficld; measurement; moasurement uncertainly; power frequency.

Accepted: April 13, 1994

\section{Introduction}

This paper reconsiders a problem related to the measurement of power frequency magnetic fields from electrical appliances using three-axis circular coil probes. Specifically, it reexamines the differ. ences between the average magnetic flux density as determined using a magnetic field meter with a three-axis circular coil probe and the magnetic flux density at the center of the probe, $B_{0}$, assuming the field is produced by a small loop of alternating current, i.e., a magnetic dipole. The "average" arises as a consequence of the averaging effects of the coil probes over their cross sectional areas when placed in a nonuniform magnetic field. The differences between the average magnetic field and $B_{0}$ can be regarded as measurement errors because the center of the probe is normally considered the measurement location. The magnetic dipole field is chosen as the relevant field because its geometry provides a good approximation of the magnetic field produced by many electrical appliances [1].
The average magnetic flux density measured by a threc-axis magnetic field meter, $B_{x \times 3}$, is also referred to as the resultant magnetic field and is defined as [2]

$$
B_{w 33}=\sqrt{B_{1}^{2}+B_{2}^{2}+B_{5}^{2}}
$$

where $B_{1}, B_{2}$, and $B_{3}$ are average root-mean-square (tms) magnetic field components determined by each of three orthogonally oriented coil probes.

Differences between $B_{2 v 3}$ and $B_{0}$ are calculated as a function of $r / a$ where $r$ is the distance between the magnetic dipole and the center of the probe, and $a$ is the radius of the three-axis probe. In addition, differences between $B_{x 3}$ and $B_{0}$ are examined for different orientations of the magnetic dipole and rotations of the three-axis probe. Because the relative orientation of the dipole and three-axis probe is not known during most measurement situations, there is a distribution of possible differences between $B_{\text {av3 }}$ and $B_{0}$, and these differences 
collectively represent a source of measurement uncertainty for a given $r / a$. What will be of interest in this paper is the largest difference that occurs between $B_{3 \times 3}$ and $B_{0}$ as a function of $r / a$ (for all possible orientations of the dipole). This largest difference is designated $\Delta B_{\text {max? }}$.

This investigation extends an earlier treatment of the problem which considered different orientations of the dipole, but not all possible orientations of the three-axis probe [3]. The maximum difference between $B_{\text {avy }}$ and $B_{0}, \Delta B_{\max 3}$, is found by a numerical search during which $B_{a x}$ is determined by numerical integration. The major advance over the earlier study is the development of an expression giving the average magnetic flux density for a circular coil probe for any position and orientation of the probe in the dipole magnetic field. This development allows the search for $\Delta B_{\max 3}$ to consider "all" possible rotations of the three-axis probe. The extended search is shown to leave unchanged the values of $\Delta B_{\max }$ that were determined by the earlier treatment.

\section{Expression for Average Magnetic Field}

In the derivation given below, it is assumed that the cross sectional areas of the wire in the coil probes and the opposing magnetic fields produced by currents induced in the probes are negligible. We also assume that the three orthogonally oriented coils of the three-axis probe have circular cross sections of equal area. These assumptions either can be met in practice or can be taken into account by a calibration process.

The average magnetic flux density, $B_{\mathrm{av}}$, for a single circular coil probe with cross sectional area $A$ is given by

$$
B_{\mathrm{av}}=\frac{1}{A} \iint_{A} B \cdot n \mathrm{~d} A,
$$

where $\mathrm{d} A$ is an element of probe area, $\boldsymbol{n}$ is a unit vector perpendicular to $A$, and $B$ is the magnetic flux density. In spherical coordinates, the magnetic flux density for a small current loop of radius $b$ is [4]

$$
B=\frac{\mu_{0} d b^{2}}{2 r^{3}} \cos \theta u_{r}+\frac{\mu_{1} / b^{2}}{4 r^{3}} \sin \theta u_{\theta},
$$

where $\mu_{0}$ is the permeability of vacuum, $I$ is the alternating current, and $\boldsymbol{u}_{r}$ and $\boldsymbol{u}_{\theta}$ are unit vectors in the directions of increasing $r$ and $\theta$, respectively. The assumption is made that $b \ll r$, and the sinu- soidal time dependence of the field has been suppressed. The value of $B_{0}$ is given by the magnitude of $B$ [Eq. (3)]. Figure 1 shows the spherical coordinates $r$ and $\theta$, a small current loop at the origin of the coordinate system, and a sketch of a three-axis probe. The center of the probe coincides with the origin of the prime coordinate system $x^{r}, y^{\prime}$, and $z^{\prime}$. The coil probes are labelled P1, P2, and P3, have unit normal vectors $\boldsymbol{n}_{1}, \boldsymbol{n}_{2}$, and $\boldsymbol{n}_{3}$, respectively, and are shown in Figure 1 (inset) for illustrative purposes as being in the directions of prime coordinates. The orientation of the magnetic dipole with respect to the position of the probe is characterized by the angle $\theta$.

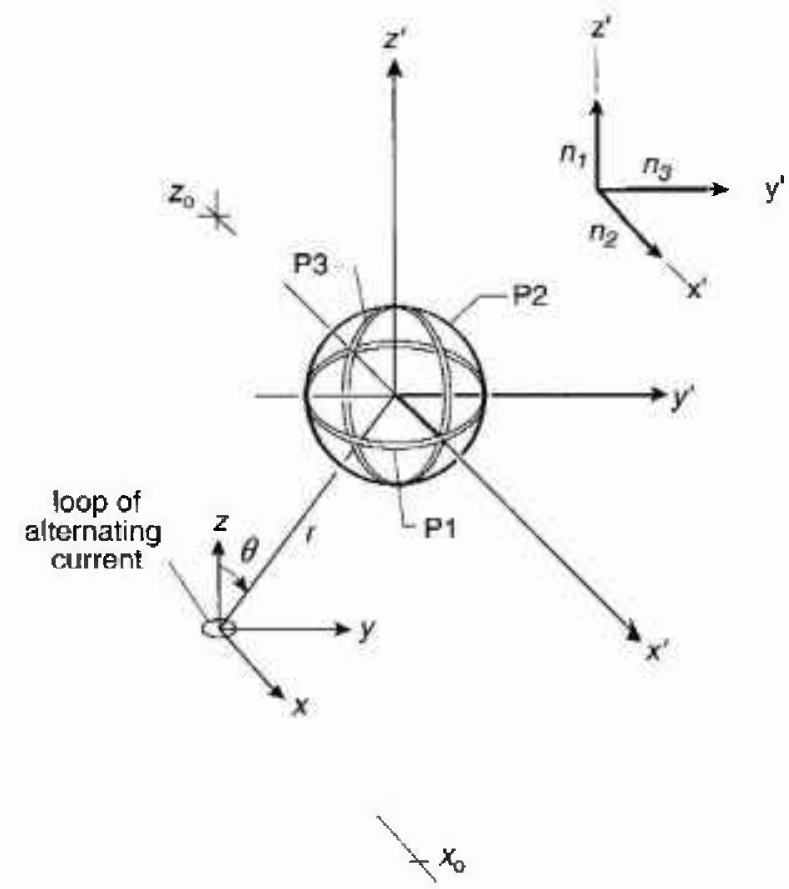

Fig. 1. Three-axis magnetic field probe with its center at $x=x_{0 h}$ $y=0$, and $z=z_{0}$. A small currcnt loop producing a dipole mag Jetic tield is located at the origin of the unprimed courdinate system. The unit vectors $n_{1}, n_{2}$, and $n_{3}$ are normal to the areas of probes $\mathrm{P} 1, \mathrm{P} 2$, and $\mathrm{P} 3$, respectively. Changes in the angle $\theta$ correspond lo varying the oricntation of the dipole with respect to the probe.

For our purposes, it is convenient to express $B$ in terms of Cartesian coordinates. The magnetic flux density is then [2]

$$
B=i \frac{3 C x z}{2 r^{5}}+j \frac{3 C y z}{2 r^{5}}+k \frac{C}{2 r^{3}}\left(\frac{3 z^{2}}{r^{2}}-1\right)
$$

where $r=\left[x^{2}+y^{2}+z^{2}\right]^{1 / 2}, i, j$, and $k$ are unit vectors for the Cartesian coordinates, and $C$ is the constant $\mu_{0} I b^{2} / 2$. 
The goal is to develop an expression for $B_{a v}$ at an arbitrary point which can be evaluated for any orientation of the coil probe. The value of $B_{a v 3}$ can then be found by combining the rms values of $B_{\mathrm{av}}$ from three orthogonal directions according to Eq. (1). The approach described below for obtaining the desired expression for $B_{\mathrm{a}}$ is to transform the problem into the coordinate system of the coil probe. In this coordinate system, the unit vector normal to the plane of the coil coincides with the " $z$-axis", $B$ is expressed in terms of the probe coordinates, and the integration over the area of the circular coil probe is carried out numerically in polar coordinates.

We begin by considering, without loss of generality, a three-axis coil probe with its center at $x=x_{i l}$, $y=0$, and $z=z_{i h}$ where $x_{i n}=r \sin \theta$ and $z_{0}=r \cos \theta$ (Fig. 1). We then focus on coil probe P1 and its unit vector $n_{t}$ after it is rotated through angles $\alpha_{1}$ and $\alpha_{2}$ with respect to the prime coordinate system as shown in Fig. 2. The unit vectors $n_{2}$ and $n_{3}$ will also change in orientation to maintain their orthogonal relationship, but are not shown for purposes of clarity. In the prime coordinate system, $n_{1}$ is given by

$$
n_{1}=i \sin \alpha_{1} \cos \alpha_{2}+j \sin \alpha_{1} \sin \alpha_{2}+k \cos \alpha_{1} .
$$

By examination,

$$
\begin{aligned}
n_{2}= & i \sin \left(\alpha_{1}+90^{\circ}\right) \cos \alpha_{2}+j \sin \left(\alpha_{1}+90^{\circ}\right) \sin \alpha_{2} \\
& +k \cos \left(\alpha_{1}+90^{\circ}\right) \\
& =i \cos \alpha_{1} \cos \alpha_{2}+j \cos \alpha_{1} \sin \alpha_{2}-k \sin \alpha_{1} .
\end{aligned}
$$

The remaining unit vector, $n_{3}$, is given by

$$
\begin{aligned}
\boldsymbol{n}_{3} & =\boldsymbol{n}_{1} \times \boldsymbol{n}_{2} \\
& =-i \sin \alpha_{2}+j \cos \alpha_{2},
\end{aligned}
$$

but it also can be deternined by examination, i.e., $\alpha_{1}$ is replaced by $90^{\circ}$ and $\alpha_{2}$ is replaced by $\alpha_{2}+90^{\circ}$ in Ex. (5). For this case, $\boldsymbol{n}_{3}$ is constrained to lie in the $x^{\prime}-y^{\prime}$ plane. Later this constraint is removed.

The coordinate system of the probe is reached by the following transformations:

(i) translation of the origin to the origin of the prime coordinates shown in Fig. 1,

(ii) rotation of the prime coordinates through angle $\alpha_{2}$ about the $z^{\prime}$-axis, yielding the doubleprime coordinates $x^{\prime \prime}, y^{\prime \prime}, z^{\prime \prime}$, as shown in Fig. 2, and

(iii) rotation of the double-prime coordinates through angle $\alpha_{1}$ about the $y^{\prime \prime}$-axis yielding the triple-prime coordinates $x^{\prime \prime \prime}, y^{\prime \prime \prime}, z^{\prime \prime \prime}$ (Fig. 2). In the triple-prime coordinate system, the normal vector, $n_{\mathrm{b}}$, given by Eq. (5), is along the $z^{m}$-axis as desired.

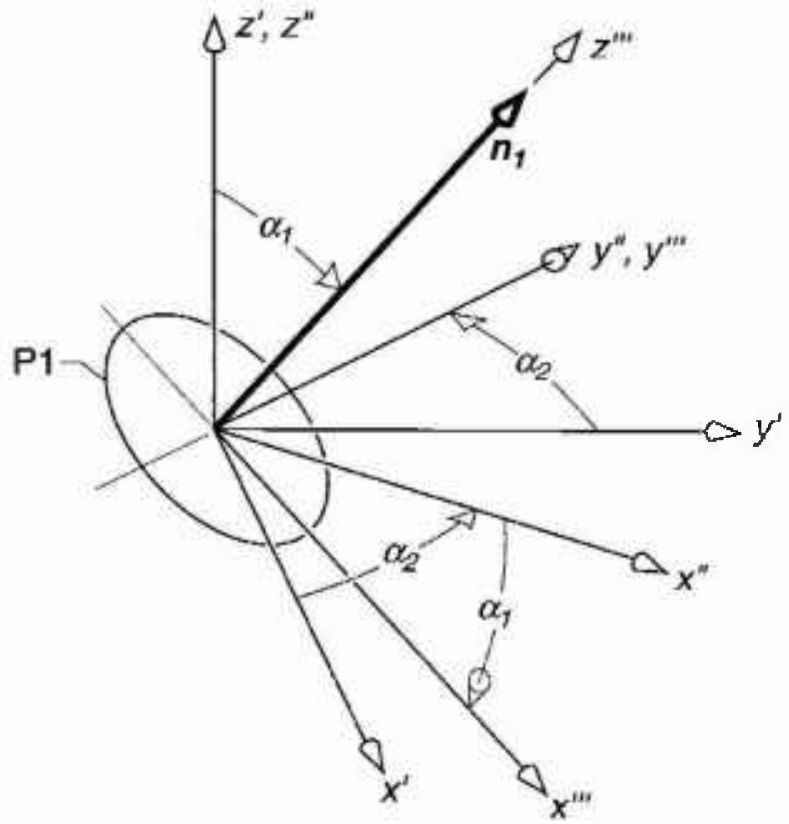

Fig. 2. (a) Geometry of unit vecior $n_{1}$ and coordinates after rotation of the prime coordinates through angle $\alpha_{2}$ about the $z^{\prime}$-axis and after rotation of double-prime coordinates through atngle $\alpha_{1}$ about $y^{\prime \prime}$-axis.

Transformation (i) is given by

$$
\begin{aligned}
& x=x_{0}+x^{\prime} \\
& y=y^{\prime} \\
& z=z_{0}+z^{\prime} .
\end{aligned}
$$

The first rotation of coordinates (ii) is given by

$$
\begin{aligned}
& x^{\prime \prime}=x^{\prime \prime} \cos \alpha_{2}-y^{\prime \prime} \sin \alpha_{2} \\
& y^{\prime \prime}=x^{\prime \prime} \sin \alpha_{2}+y^{\prime \prime} \cos \alpha_{2} \\
& z^{\prime}=z^{\prime \prime},
\end{aligned}
$$

and the second rotation (iii) is given by

$$
\begin{aligned}
& z^{\prime \prime}=z^{m} \cos \alpha_{1}-x^{m} \sin \alpha_{1} \\
& x^{n}=z^{\prime \prime \prime} \sin \alpha_{1}+x^{m \prime} \cos \alpha_{1} \\
& y^{\prime \prime}=y^{\prime \prime} .
\end{aligned}
$$

From Eqs. (8)-(10), we have

$$
\begin{aligned}
& x=x_{1}+\left(z^{\prime \prime \prime} \sin \alpha_{1}+x^{\prime \prime \prime} \cos \alpha_{1}\right) \cos \alpha_{2}-y " ' \sin \alpha_{2} \\
& y=\left(z^{\prime \prime \prime} \sin \alpha_{1}+x^{\prime \prime \prime} \cos \alpha_{1}\right) \sin \alpha_{2}+y^{\prime \prime \prime} \cos \alpha_{2} \\
& z=z_{0}+z^{\prime \prime} \cos \alpha_{1}-x^{\prime \prime} \sin \alpha_{1},
\end{aligned}
$$

which, when substituted into $E q$. (4), expresses $B$ in terms of the probe coordinates. A simplification of Eq. (11) is had by noting that the integration over the area of the probe [Eq. (2)] occurs in the $x^{m \prime \prime}-y^{m}$ plane, i.e., $z^{\prime \prime}=0$. Contributions to $B_{a ; 3}$ from each 
of the coil probes is found by using the appropriate normal vector [Eqs. (5)-(7)] during the integrations. As noted above, the integration is carried out numerically (using a double Simpson's Rule) in polar coordinates, i.e.,

$$
\begin{aligned}
& x^{\prime \prime \prime}=\rho \cos \psi, \quad 0 \leqslant \rho \leqslant a, \quad 0 \leqslant \psi<2 \pi \\
& y^{\prime \prime \prime}=\rho \sin \psi, \\
& \mathrm{d} A=\mathrm{d} x^{\prime \prime \prime} \mathrm{d} y^{\prime \prime \prime}=\rho \mathrm{d} \rho \mathrm{d} \psi .
\end{aligned}
$$

The accuracy of the numerical integrations was checked by increasing the number of divisions between the limits of integration for $\rho$ and $\psi$. The results reported below were not affected by further refinements of the intervals used during the integrations.

In the search for $\Delta B_{\text {max3, }}$ it will be necessary to perform rotations of the three-axis probe about the $z^{m}$-axis or $n_{1}$ direction (see Search Protocol below), i.e., the unit vectors $n_{2}$ and $n_{3}$ for probes $P 2$ and P3 are rotated about $n_{1}$. This removes the constraint noted earlier that $n_{3}$ lies in the $x^{\prime}-y^{\prime}$ plane. Because the integrand for $B_{a v}$ is in terms of the $\alpha$ angles, relationships must be found between the angle of rotation about the $z^{\prime \prime}$-axis, designated as $\phi$, and the $\alpha$ values that appear in the integrands for $\mathrm{P} 2$ and $P 3$. These relationships are found by examining the unit vectors for the probes $n_{2}$ and $n_{3}$ as they rotate about the $z^{m-a x i s ~ o r ~} n_{1}$ direction.

In Figs. 3 and 4, consider $n_{1}$ in a direction characterized by the angles $\alpha_{10}$ and $\alpha_{20}$, i.e.,

$$
n_{1}=i \sin \alpha_{10} \cos \alpha_{20}+j \sin \alpha_{10} \sin \alpha_{20}+k \cos \alpha_{10}
$$

From Eqs. (6) and (7), we have

$$
\begin{aligned}
\boldsymbol{n}_{2}= & i \sin \left(\alpha_{10}+90^{\circ}\right) \cos \alpha_{10}+j \sin \left(\alpha_{10}+90^{\circ}\right) \sin \alpha_{20} \\
& +k \cos \left(\alpha_{10}+90^{\circ}\right)
\end{aligned}
$$

and

$$
\begin{aligned}
n_{3}= & i \sin \left(90^{\circ}\right) \cos \left(\alpha_{21}+90^{\circ}\right)+j \sin \left(90^{\circ}\right) \sin \left(\alpha_{20}+90^{\circ}\right) \\
& +k \cos \left(90^{\circ}\right) .
\end{aligned}
$$

The trigonometric expressions in Eqs. (14) and (15) are not simplified in order to aid the reader in seeing the relationships between the three unit vectors.

Following a counterclockwise rotation of $\phi$ degrees about the $z^{\text {th }}$-axis, the $\alpha_{2}$ 's will increase in value and the $\alpha_{1}$ 's will decrease in value in the expressions for $\boldsymbol{n}_{2}$ and $\boldsymbol{n}_{3}$. These changes also occur in the expression for the magnetic flux density $B$.

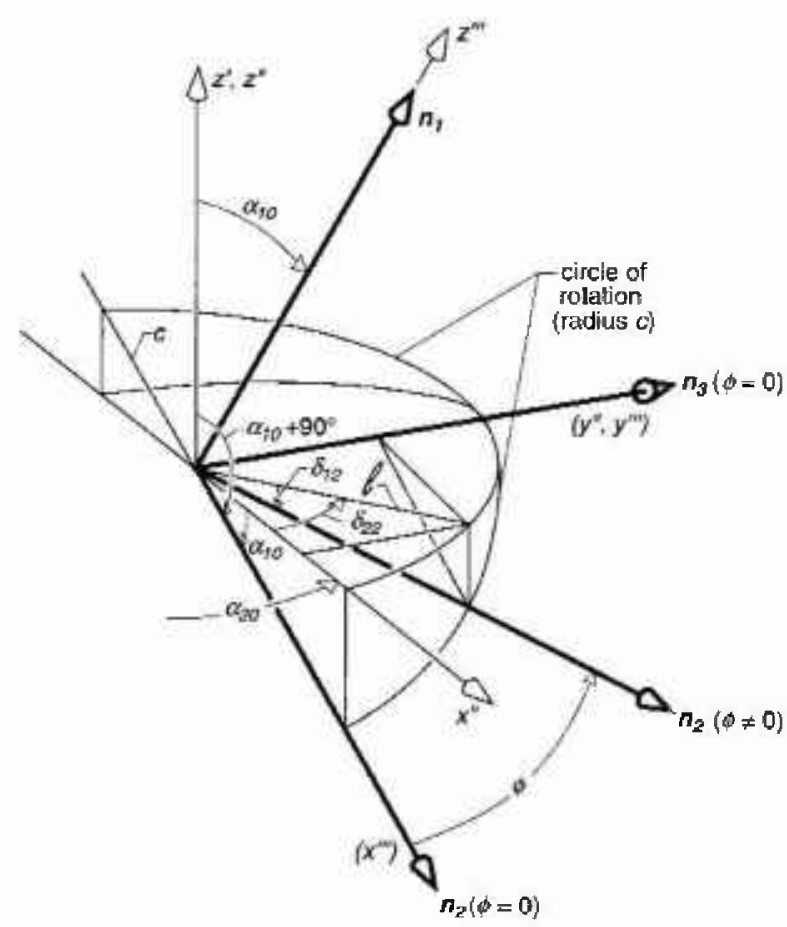

Ejg. 3. Gcometry of coordinates and unit vectors after unit vector $n_{2}$ is rotated $\phi$ degrees about $n_{1}$ or $z^{m \prime}$-axis. The rotation of $n_{7}$ is not shown for purposes of clarity (sec Fig. 4).

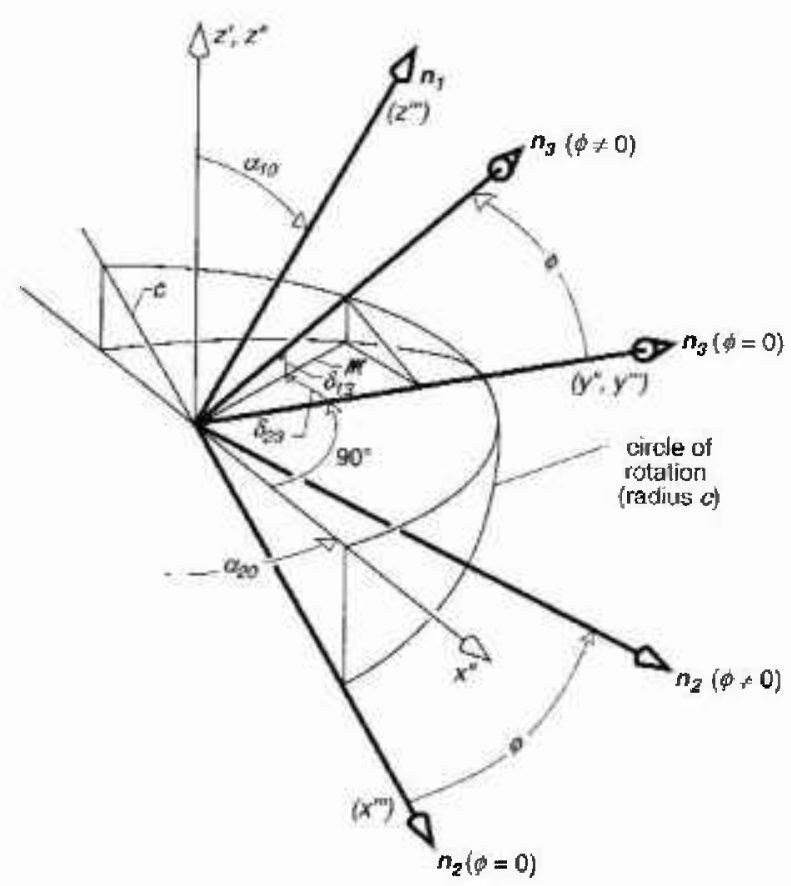

Fig. 4. Geometry of coordinates and unit vectors after unit vectors $n_{3}$ and $n_{2}$ are motated through angle $\phi$ about $n_{1}$ or $z^{\prime \prime}$-axis. 
After a rotation of $\phi$ degrees, a line along the unit vector $n_{2}$ will intersect the circle of rotation in the $x^{m \prime \prime}-y^{\prime \prime \prime}$ plane at a point given by (Fig. 3)

$$
\begin{aligned}
& x^{m+}=c \cos \phi \\
& y^{n t}=c \sin \phi \\
& z^{n+t}=0,
\end{aligned}
$$

where the radius for the rotation has been arbitrarily taken to be some constant c. From Eqs. (10) and (16), the same point in the double prime coordinate system is

$$
\begin{aligned}
& z^{\prime \prime}=-x^{m p} \sin \alpha_{10}=-c \cos \phi \sin \alpha_{10} \\
& x^{\prime \prime}=x^{m+1} \cos \alpha_{10}=c \cos \phi \cos \alpha_{10} \\
& y^{\prime \prime}=y^{n+}=c \sin \phi .
\end{aligned}
$$

The increment to $\alpha_{20}$ for unit vector $\boldsymbol{n}_{2}$ after rotation $\phi, \delta_{22}$ (Fig. 3), can be found from the expression for its tangent, i.e.,

$$
\begin{aligned}
\tan \delta_{22} & =\frac{y^{\prime \prime}}{x^{\prime \prime}}=\frac{\tan \phi}{\cos \alpha_{10}} \\
\delta_{22} & =\tan ^{-1}\left(\frac{\tan \phi}{\cos \alpha_{101}}\right) .
\end{aligned}
$$

Prior to the rotation, the angle with respect to the $z^{\prime}$-axis for the unit vector $n_{2}$ is given by $\alpha_{10}+90^{\circ}$ (Fig, 3). After the rotation, the corresponding angle will be $\delta_{12}+90^{\circ}$ where $\delta_{12}<\alpha_{10}$. The value of $\delta_{12}$ is found by noting that after the rotation $\phi$, the line $l$ from the origin to the projection of the circle of rotation onto the $x^{\prime \prime}-y^{\prime \prime}$ plane is given by

$$
l=\sqrt{\left(x^{\prime \prime}\right)^{2}+\left(y^{\prime \prime}\right)^{2}}
$$

and that the tangent for $\delta_{[2}$ is just $\left|z^{\prime \prime} / l\right|$ (Fig.3). From Eqs. (17) and (19),

$$
\delta_{12}=\tan ^{-1}\left|\frac{z^{\prime \prime}}{l}\right|=\tan ^{-1}\left(\frac{\cos \phi \sin \alpha_{10}}{\sqrt{\cos ^{2} \phi \cos ^{2} \alpha_{10}+\sin ^{2} \phi}}\right) \text {. }
$$

Thus, following a rotation of $\phi$ degrees about $\boldsymbol{n}_{1}$, $\alpha_{20}$ and $\alpha_{10}+90^{\circ}$ will be replaced by $\alpha_{20}+\delta_{22}$ and $\delta_{12}+90^{\circ}$, respectively, in the expressions for $\boldsymbol{n}_{2}$ and $B$ during the calculation of $B_{w}$ for probe $\mathrm{P} 2$.

The $\alpha$ values for $n_{3}$ can be determined with a similar analysis. Following a rotation of $\phi$ degrees about the $z^{m \prime}$-axis (Fig. 4), a line along the unit vector $n_{3}$ will intersect the circle of rotation in the $x^{m}-y^{m}$ plane at the point

$$
\begin{aligned}
& x^{m+1}=-c \sin \phi \\
& y^{m \prime}=c \cos \phi \\
& z^{m \prime}=0 .
\end{aligned}
$$

From Eqs. (10) and (21), the same point in the double prime coordinate system is

$$
\begin{aligned}
& z^{\prime \prime}=c \sin \phi \sin \alpha_{! \prime} \\
& x^{\prime \prime}=-c \sin \phi \cos \alpha_{10} \\
& y^{\prime \prime}=c \cos \phi .
\end{aligned}
$$

Following rotation $\phi$, the angle $\alpha_{20}+90^{\circ}$ for $n_{3}$ [Eq. (15)] will increase by an amount $\delta_{2,}$ as shown in Fig. 4. The increment, $\delta_{23}$, can be found from the expression for the absolute value of its tangent, i.e.,

$$
\begin{aligned}
\tan \delta_{y s} & =\left|\frac{x^{n}}{y^{n}}\right|=\tan \phi \cos \alpha_{10}, \\
\delta_{23} & =\tan ^{-1}\left(\tan \phi \cos \alpha_{10}\right) .
\end{aligned}
$$

Prior to the rotation, the unit vector $n_{3}$ makes an angle of $90^{\circ}$ with respect to the $z^{\prime}$-axis (Fig. 4). After the rotation, this angle will decrease by an amount $\delta_{13}$. The value of $\delta_{13}$ is found by noting that after the rotation $\phi$, the line $m$ from the origin to the projection of the circle of rotation onto the $x^{\prime \prime}-y^{\prime \prime}$ plane is given by

$$
m=\sqrt{\left(x^{\prime \prime}\right)^{2}+\left(y^{\prime \prime}\right)^{2}}
$$

and that the tangent for $\delta_{13}$ is just $z^{\prime \prime} / m$ (Fig. 4).

From Eqs. (22) and (24) we have

$$
\begin{aligned}
\tan \delta_{13} & =\frac{z^{\prime \prime}}{m}=\frac{\sin \phi \sin \alpha_{113}}{\sqrt{\sin ^{2} \phi \cos ^{2} \alpha_{10}+\cos ^{2} \phi}} \\
\delta_{13} & =\tan ^{-1}\left(\frac{\sin \phi \sin \alpha_{10}}{\sqrt{\sin ^{2} \phi \cos ^{2} \alpha_{10}+\cos ^{2} \phi}}\right) .
\end{aligned}
$$

Thus, following a rotation of $\phi$ degrees about $n_{1}$, $\alpha_{20}+90^{\circ}$ and $90^{\circ}$ are replaced by $\alpha_{30}+90^{\circ}+\delta_{23}$ and $90^{\circ} \delta_{1,3}$, respectively in the expressions for $n_{3}$ [Eq. (15)] and $B$ during calculation of $B_{\mathrm{av}}$ for coil probe P3.

\section{Search Protocol}

The search for the largest difference between $B_{\text {avs }}$ and $B_{0}, \Delta B_{\max }$, for a given distance $r$ from the dipole proceeds as follows:

(i) For a fixed distance $r$ away from the dipole, and with $\theta=\alpha_{1}=\alpha_{2}=0$, the three-axis probe is rotated about the $z^{m}$-axis or $n_{1}$ direction in $2^{\circ}$ steps (i.e., $\phi$ is incremented in $2^{\circ}$ steps). Then $B_{x w}$ for each coil probe is evaluated and combined according to Eq. (1) for each value of $\phi$ to obtain $B_{a v 3,} B_{3 v 3}$ is compared with $B_{b,}$ and the largest difference is 
saved. Because of the symmetry of the problem, a total rotation of $90^{\circ}$ is required to cover all the cases (with $2^{\circ}$ increments).

(ii) The angle $\alpha_{1}$ is advanced in $5^{\circ}$ steps and the above comparisons are repeated as the probe is rotated about the $z^{m}$-axis or $n_{1}$ direction. The maximum value of $\alpha_{1}$, without duplication of results is $90^{\circ}$.

(iii) For each value of $\alpha_{1}, \alpha_{2}$ is incremented from $0^{\circ}$ in steps of $5^{\circ}$ and the above comparisons are repeated. Because of symmetry arguments, a total rotation of $180^{\circ}$ is required to consider all the cases without duplication.

(iv) Following the above calculations, different orientations of the magnetic dipole are considered by changing the angle $\theta$ in $15^{\circ}$ steps and repeating steps (i) through (iii). The choices of increments indicated above were found to provide adequate sensitivity for determining $\Delta B_{\text {arz. }}$.

(v) Steps (i) through (iv) are repeated for different values of $r$.

A diagram schematically indicating several positions for $n_{1}$, and rotations about $n_{1}$, as the above protocol was carried out for a fixed value of $r$ is shown in Fig. 5.

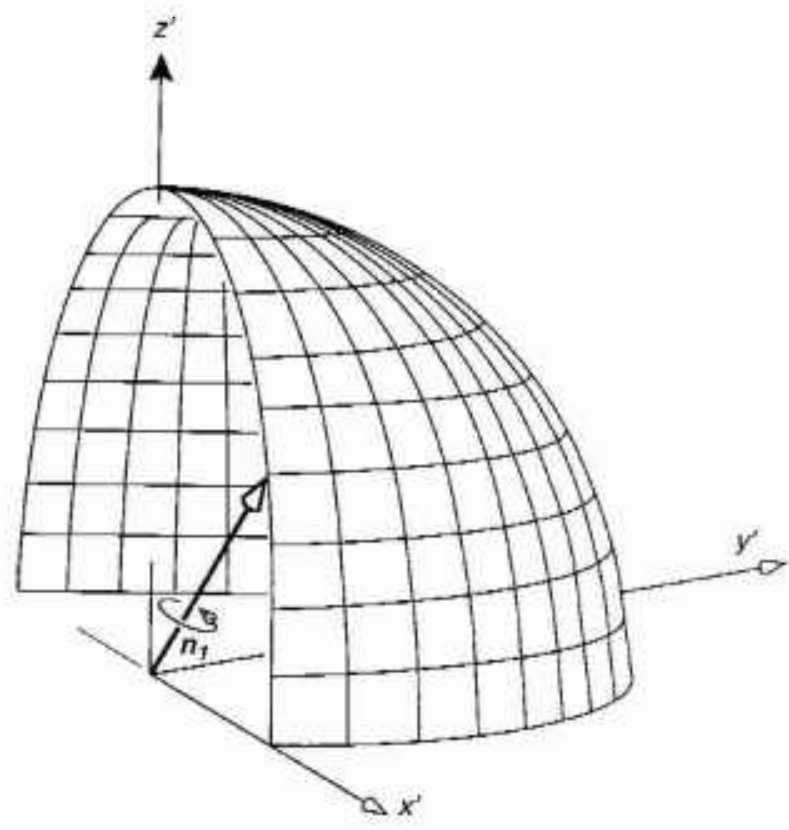

Fig. 5. Trajectorics of $n_{1}$ during search for $\Delta B_{\text {max }}$. The unil vectors $n_{2}$ and $n_{3}$ are not shown but maintain their orthogonal relationship wilh $n_{1}$ throughout the search.

\section{Results and Discussion}

As already noted, an earlier search for $\Delta B_{\text {max }}$ [3] was not as comprehensive as the one described in this paper. While the ratio $r / a$ and $\theta$ could be varied without restriction, the rotations of the three-axis probe were limited to simple rotations about the $x^{\prime}-, y^{\prime}-$, or $z^{\prime}$-axis. That is, it was not possible to consider differences between $B_{\text {av } 3}$ and $B_{0}$ for combinations of rotations about two or three axes. This problem has been overcome with a more generalized expression for $B_{\text {av }}$ compared to the ones used in the earlier calculation. What is perhaps surprising, however, is that the $\Delta B_{\text {mas }}$ values obtained with the more comprehensive search protocol are the same as previously calculated. That is, $\Delta B_{\max }$ is negative and occurs for all $r / a$ values when $\theta=90^{\circ}$, as previously found, and correspond to the $\Delta B_{\max }$ values determined earlier following simple rotations about the $y^{s}$-axis (referred to as " $\alpha$ rotations" in Ref. [3]). Numerical values of $\Delta B_{\max 3}$ are provided in Table 1 as a function of $r / a$.

Table 1. Values of $\Delta B_{\max 3,}$ as a function of normalized distance ria from magnetic dipole

\begin{tabular}{rr}
\hline \hline$r / a$ & $\Delta B_{\max 3}(\%)$ \\
\hline 3 & -19.6 \\
4 & -10.8 \\
5 & -6.9 \\
6 & -4.8 \\
7 & -3.5 \\
8 & -2.7 \\
9 & -2.1 \\
10 & -1.7 \\
11 & -1.4 \\
12 & -1.2 \\
13 & -1.0 \\
14 & -0.9 \\
15 & -0.8 \\
\hline
\end{tabular}

\section{Conclusions}

The present calculations have determined the largest differences between the resultant magnetic field, $B_{\mathrm{av}}$, and the field value at the center of the probe $B_{v}$, assuming a dipole magnetic field. These largest differences, designated $\Delta B_{\text {max }}$, are reported in Table 1 as a function of normalized distance, $r / a$, from the center of the dipole and agree with values previously found after a far less comprehensive search [3]. The quantity, $\Delta B_{\max }$, can be regarded as the largest error due to instrumental 
averaging effects. As noted earlier, because the relative orientations of the dipole and three-axis probe are not known for a given r/a under typical measurement conditions, there will be a range of possible differences between $B_{\text {av } 3}$ and $B_{0}$. Thus, ideally, it would be desirable to determine the distribution of differences between $B_{\mathrm{av} 3}$ and $B_{v}$ and treat the problem using a statistical approaçh, but that has been left to a future calculation.

Because the dipole field is a good approximation of fields produced by many electrical appliances, the information in Table 1 should be taken into account when total uncertainties are being determined during measurements of magnetic fields from appliances. For example, if the resultant magnetic field is to be measured at a distance $r$ from an appliance with a combined relative standard uncertainty [5] of less than $\pm 10 \%$, magnetic field meters with three-axis probes having radii $a$ such that $r / a \approx 3$ should be considered unsuitable. Three-axis probes having radii such that $r / a=5$ would conservatively be considered suitable if the combined relative standard uncertainty from all other sources (e.g., calibration process, frequency response) amounted to about $3 \%$ or less, since $6.9 \%+$ $3.0 \%=9.9 \%$, where $6.9 \%$ is taken from Table 1 for $r / a=5$.

\section{Acknowledgments}

The authors are pleased to acknowledge the assistance of Edward Kelley in enhancing the speed of the computer program used for the calculations. Support was received from the Office of Energy Management of the U.S. Department of Energy.

\section{References}

[1] D. L. Mader and S. B. Peralta, Residential Exposure to 60Hz Magnetic Fields From Appliances, Bioelectromagnetics 13, 287-301 (1992).

[2] JEEE Magnetic Fields Task Force, A Protocol for Spot Measurements of Residential Power Frequency Magnetic Fields, IEEE Trans. Power Delivery 8, 1386-1394 (1993)-

[3] M. Misakian, Coil Probe Dimensions and Uncertaintics During Mcasurcments of Nonuniform ELF Magnetic Fields, J. Res. Natl. Inst. Stand. Technol. 98, 287-295 (1993).

[4] D. Corson and P. Lorrain, Introduction to Electromagnetic Fields and Waves, W. H. Freeman, San Francisco, CA (1962) p. 210.

[5] B. N. Taylor and C. E. Kuyatt, Guidelines for Evaluating and Expressing the Uncertainty of NIST Measurement Results, NIST Technical Note 1297 (1993).
About the authors: $M$. Misakian is a physicist and $C$. Fenimore is a mathematician in the Electricity Division of NIST. The National Institute of Standards and Technology is an agency of the Technology Administration, U.S. Deparment of Commerce. 